\title{
Collaboration on a public health-driven vaccine initiative
}

\author{
Desai $\mathrm{S}^{1 *}$, Tsang $\mathrm{R}^{2}$, St. Laurent $\mathrm{M}^{1}$, Cox $\mathrm{A}^{3}$ \\ ${ }^{1}$ Center for Immunization and Respiratory Infectious Diseases, Public Health Agency of Canada, Ottawa, ON \\ ${ }^{2}$ National Microbiology Laboratory, Public Health Agency of Canada, Winnipeg, MB \\ ${ }^{3}$ Vaccine Program, Human Health Therapeutics, National Research Council of Canada, Ottawa, ON \\ * Corresponding author: shalini.desai@oahpp.ca
}

\section{Abstract}

Disease surveillance can be used as an opportunity to determine priorities for research and the development of new therapeutics. This is evident in the work underway to develop a new vaccine to combat a serious invasive childhood disease: Haemophilus influenzae serotype a (Hia). Following the introduction of Hib vaccine into the routine childhood immunization schedule in Canada in the early 1990's, the Public Health Agency of Canada (PHAC) began to document the dropping rates of $\mathrm{H}$ influenzae serotype b (Hib) infection. However, invasive $H$. influenzae diseases due to non-Hib strains began to increase and in 2007, surveillance for invasive $H$. influenzae disease due to all serotypes as well as non-typeable strains was initiated. Current data suggests Hia is a cause of serious invasive disease, particularly in Aboriginal populations. Similar to Hib, Hia causes severe illnesses such as meningitis, sepsis and bacteremic pneumonia in young children under the age of five.

Given the emerging threat due to Hia in Aboriginal populations in Canada, PHAC formed a partnership with the National Research Council of Canada (NRC) to investigate the potential of creating a capsular polysaccharide vaccine against Hia. At the present time, candidate vaccine seed strains have been identified and PHAC and the NRC are working with the Northern Ontario School of Medicine, the US Centers for Disease Control and Prevention and others. The goal of this research is to identify and prepare a candidate vaccine against Hia while increasing the understanding of how such a vaccine would improve the health of a vulnerable population.

\section{Introduction}

One of the benefits of disease surveillance is that it can highlight the need or opportunity for new interventions. Surveillance provides an essential means of identifying diseases that create a significant burden within a population and helps to identify the need for vaccines, one of the most effective public health interventions to prevent disease. For example vaccines against smallpox have eradicated this disease (1) and polio has been eliminated from almost all countries of the world (2). As a result of surveillance data, vaccines have been created to prevent diseases such as Streptococcus pneumoniae, Neisseria meningitides as well as influenza.

As part of its role to serve the public good, through surveillance, the public health sector can identify the need for and facilitate the development of new vaccines against diseases that cause significant morbidity and mortality in the population.

This article outlines a public health-driven collaboration that has emerged between the Public Health Agency of Canada (PHAC, or the Agency) and the National Research Council of Canada (NRC) to develop a vaccine that combats an emerging pathogen particularly prevalent within the Aboriginal population: Haemophilus influenzae serotype a (Hia).

\section{The Public Health Agency of Canada}

The Public Health Agency of Canada was created in 2004 to provide federal leadership to anticipate, respond and be accountable for public health issues and emergencies (3). The Agency was also envisioned as a means to improve collaboration between jurisdictions to protect and promote public health within the Canadian population. 
Within the Agency, the Centre for Immunization and Infectious Respiratory Diseases (CIRID) (4) and the National Microbiology Laboratory (NML) (5) are primarily responsible for providing technical support for vaccinepreventable diseases. CIRID is responsible for epidemiological surveillance of many vaccine-preventable diseases, while NML provides laboratory surveillance, reference diagnostics and research. In cooperation, they provide data on emerging trends for a number of infectious diseases.

\section{The National Research Council of Canada}

The NRC began its work to develop vaccine technologies during the 1980's. Its area of expertise has developed over the years to include excellence in carbohydrate chemistry, immunochemistry and in developing partnerships to create new vaccines $(6,7,8)$. The NRC has also developed expertise to improve current vaccines and technologies that enable ongoing vaccine innovation. Through the NRC, there are technical and advisory services, research facilities, licensing opportunities as well as program and partnership opportunities. This expertise has been applied to study carbohydrate antigens of encapsulated bacterial pathogens such as Haemophilus influenzae serotype b (9), Neisseria meningitidis $(6,7)$ and streptococci $(10)$, which are important pathogens. Each of these pathogens has a polysaccharide capsule that enhances its virulence. In recent years, the NRC has focused on antigen selection, glycoconjugation technologies, adjuvant design and production of vaccine candidates all compatible with current Good Manufacturing Practices (cGMP). The NRC also has the capability to conduct pre-clinical in vivo trials to monitor efficacy and safety of products produced (11).

\section{Invasive Haemophilus influenzae disease in Canada}

Prior to the development of a $H$. influenzae serotype b (Hib) conjugate vaccine, Hib was a significant cause of meningitis, sepsis and bacteremic pneumonia in children under the age of five years. Based on national surveillance from $1986-87$, approximately 700 cases of invasive Hib disease occurred per year. Hib vaccines were introduced into the routine childhood immunization schedule in Canada in the early 1990's. Since vaccine introduction, the incidence of Hib disease has dramatically declined. In the last five years where data is available at a national level (2008 - 2012), there has been an average of 26 cases of Hib disease reported across Canada per year (12). However, based on data from national surveillance programs, invasive $H$. influenzae diseases due to non-Hib strains have emerged in the last decade (13). Therefore, in 2007, a revised national case definition of invasive $H$. influenzae disease to include diseases due to all serotypes as well as non-typeable strains was endorsed (14).

Among the encapsulated serotypable $H$. influenzae strains, serotype $b$ has been determined to be the most virulent followed by Hia and then others (15). Current data suggests $\mathrm{H}$. influenzae serotype a (Hia) is a cause of serious invasive disease, particularly in the Aboriginal population (16). Similar to Hib, Hia causes severe illness such as meningitis, sepsis and bacteremic pneumonia in young children under the age of five. Since northern Canada has a relatively high indigenous population and Hia, being similar to Hib including its epidemiology, has replaced $\mathrm{Hib}$ as a cause of significant burden of invasive diseases in the post-Hib vaccine era (17). This trend has also been observed in Alaska, U.S.A. (18).

\section{Public health-driven collaboration}

Given the knowledge of an emerging threat due to Hia in Aboriginal populations in Canada and with the launch of a Vaccine Program within the NRC's Human Health Therapeutics portfolio, PHAC partnered with the NRC to investigate the potential to create a capsular polysaccharide vaccine against Hia for protection of Canadians. The initial meeting and consultation between PHAC and the NRC was held in 2011 to discuss the feasibility of developing a glycoconjugate vaccine against Hia. In October 2011, a workshop organized by PHAC, the NRC and the Northern Ontario School of Medicine (NOSM) was held at Lakehead University. This workshop included academic researchers from the NOSM and the Thunder Bay Regional Health Authority who discussed two neglected infectious diseases in Aboriginal communities (Hia and Helicobacter pylori) (19). Based on the groundwork from the initial meetings, a letter of intent on "Expanding Vaccine Development in Canada" was 
signed between PHAC and the NRC in September 2012 and the joint Hia project was launched in March 2013. In December 2013, investigators from the Arctic Investigators Program of the US Centers for Disease Control and Prevention (CDC) joined the group as they had also observed a similar increase in Hia incidence within the Alaskan Aboriginal population.

At this point in time, candidate vaccine seed strains have been identified and are being characterized at the NML. Large-scale preparation of glycoconjugates based upon the capsular polysaccharide from Hia is now being carried out at the NRC. Serological assays including tests to measure protective immunity are being developed at the NOSM. Immunogenicity studies in animals that have received the glycoconjugates are being conducted at the NRC and sera derived from immunised animals are being evaluated against a range of clinical strains at NOSM.

The goal of this research is to identify and prepare a candidate vaccine against Hia while advancing understanding of how such a vaccine would improve health in a vulnerable population. Work to date has focused on how to provide a better understanding and characterization of the epidemiology of invasive Hia disease through improved surveillance activities. This is being done in collaboration with international partners such as the $\mathrm{CDC}$ and the Pan American Health Organization (PAHO). Additionally, on the laboratory side, this work will provide a proof of concept that capsular polysaccharide from Hia can be purified and conjugated to a carrier protein that will induce a protective immune response in experimental animals. Once a candidate vaccine has been shown to be safe and effective in pre-clinical trials, further production would require industry or other third party engagement.

\section{Conclusion}

This collaborative project is an excellent example of how government leadership can be effective in addressing a public health need. It also shows how identifying a Canadian research agenda can be expanded and applied internationally. Through surveillance, PHAC has the ability to identify opportunities for new vaccine strategies. The NRC has the technical expertise to develop and produce vaccine candidates. Together with additional partners such as academia and other governmental and non-governmental organizations, this model could lead to further vaccine innovation.

\section{Acknowledgements}

The authors would like to thank John Spika, Jim Richards, Marina Ulanova and Luis Barreto for their work in bringing together this collaborative project.

\section{Conflict of interest}

There are no conflicts of interest to declare.

\section{Funding}

This work was supported by the Public Health Agency of Canada and the National Research Council of Canada.

\section{References}

(1) World Health Organization. [Internet]. Geneva, Switzerland: World Health Organization [cited Sept 15, 2014]. http://www.who.int/csr/disease/smallpox/en/

(2) World Health Organization. [Internet]. Geneva, Switzerland: World Health Organization [updated Sept 9, 2014; cited Sept 15, 2014]. http://www.polioeradication.org/Dataandmonitoring.aspx

(3) Public Health Agency of Canada. [Internet]. Ottawa, Canada: Public Health Agency of Canada [updated 2011 Jun 16; cited Aug 20, 2014]. http://www.phac-aspc.gc.ca/about_apropos/what-eng.php

(4) Public Health Agency of Canada. Centre for Immunization and Respiratory Infectious Diseases (CIRID) [Internet]. Ottawa, Canada: Public Health Agency of Canada [updated 2012 Jul 23; cited 2014 Aug 20]. http://www.phacaspc.gc.ca/irid-diir/index-eng.php 
(5) Public Health Agency of Canada. National Microbiology Laboratory (NML) [Internet]. Ottawa, Canada: Public Health Agency of Canada [updated 2014 Jul 8; cited 2014 Aug 20]. https://www.nml-Inm.gc.ca/overview-apercu-eng.htm!

(6) Jennings HJ, Lugowski C, Ashton FE. Conjugation of meningococcal lipopolysaccharide R-type oligosaccharides to tetanus toxoid as route to a potential vaccine against group B Neisseria meningitidis. Infect Immun. 1984 Jan; 43(1):407-12.

(7) Jennings HJ, Lugowski C. Immunochemistry of groups A, B, and C meningococcal polysaccharide-tetanus toxoid conjugates. J Immunol. 1981 Sep; 127(3):1011-8.

(8) Jennings HJ, Lugowski C, inventors; National Research Council of Canada, assignee. Immunogenic polysaccharideprotein conjugates. United States patent US4356170A. 1982 Oct 26.

(9) Jennings HJ. Capsular polysaccharides as human vaccines. Adv Carbohydr Chem Biochem. 1983; 41:155-208.

(10) Kasper DL, Paoletti LC, Wessels MR, Guttormsen HK, Carey VJ, Jennings HJ, Baker CJ. Immune response to type III group B streptococcal polysaccharide-tetanus toxoid conjugate vaccine. J Clin Invest. 1996 Nov 15; 98(10):2308-14.

(11) National Research Council. About NRC [Internet]. Ottawa, Canada: National Research Council [updated 2014 Jul 15; cited 2014 Aug 20]. http://www.nrc-cnrc.gc.ca/eng/about/index.html

(12) Public Health Agency of Canada. Notifiable Diseases On-Line. [Internet] Ottawa, Canada. Public Health Agency of Canada. [updated 2014 Mar 31 ; cited 2014 Aug 3] http://dsol-smed.phac-aspc.gc.ca/dsol-smed/ndis/charts.php?c=pl

(13) Tsang R. Changing epidemiology of invasive Haemophilus influenzae disease. Lancet Infectious Diseases, 2008; 8:737.

(14) Public Health Agency of Canada. Case definitions for communicable diseases under national surveillance. Can Commun Dis Rep. 2014 Aug 20; 35(Suppl 2):1-40.

(15) Zwahlen A, Kroll JS, Rubin LG, Moxon R. The molecular basis of pathogenicity in Haemophilus influenzae: comparative virulence of genetically related capsular transformants and correlation with changes of the capsulation locus cap.Microbial Pathogenesis. 1989; 7:225-235.

(16) Ulanova M, Tsang RSW. Haemophilus influenzae serotype a as a cause of serious invasive infections. The Lancet Infectious Diseases. 2004; 14:70-82.

(17) Rotondo J, Sherrard L, Helferty M, Tsang R, Desai S. The epidemiology of invasive disease due to Haemophilus influenza serotype $a$ in the Canadian North from 2000 to 2010. Int J circumpolar Health. 2013; 72:1-5

(18) Bruce MG, Zulz T, DeByle C, Singleton R, Hurlburt D, Bruden D, Rudolph K, Hennessy T, Klejka J, Wenger JD. Haemophilus influenzae serotype a invasive disease, Alaska, USA, 1983-2011. Emerg Infect Dis. 2013 Jun; 19(6):9327.

(19) Ulanova M, Tsang R, Altman E. Neglected infectious diseases in Aboriginal communities: Haemophilus influenzae serotype a and Helicobacter pylori. Vaccine. 2012 Nov 19; 30(49):6960-6. 